Review Article

\title{
Coronavirus disease 2019: an overview
}

\author{
Ajna Prahalad, Varun Soti* \\ Lake Erie College of Osteopathic Medicine, Elmira, New York, USA \\ Received: 31 July 2021 \\ Accepted: 07 September 2021 \\ *Correspondence: \\ Dr. Varun Soti, \\ E-mail: vsoti@lecom.edu \\ Copyright: (C) the author(s), publisher and licensee Medip Academy. This is an open-access article distributed under \\ the terms of the Creative Commons Attribution Non-Commercial License, which permits unrestricted non-commercial \\ use, distribution, and reproduction in any medium, provided the original work is properly cited.
}

\begin{abstract}
Coronavirus disease 2019 (COVID-19) has caused over four million deaths worldwide and continues to affect millions across the globe. This review aims to provide an overview of severe acute respiratory syndrome coronavirus 2 (SARSCoV-2) and the progress made to counter COVID-19 through therapeutics and immunizations. We searched preclinical and clinical COVID-19 literature published between January 2018 through June 2021 on PubMed. The medical subject headings used in the literature search were COVID-19, SARS-CoV-2, Treatment, Remdesivir, Cytokine Storm, Hydroxychloroquine, Epidemiology, Angiotensin-Converting Enzyme 2 Receptor, Vaccines, and Mitigation. There is credible evidence that shows that the spike protein in the SARS-CoV-2's structure is crucial for the host to be infected. It spreads through different modes, primarily through aerosols and carriers. Pathophysiologically, SARS-CoV-2 triggers a cytokine storm leading to systemic inflammation, which can be fatal. Although treatment with Remdesivir and Dexamethasone and massive vaccinations have significantly reduced the mortality rate, COVID-19 remains a significant threat. The emergence of SARS-CoV-2's different strains, notably the Delta variant, demonstrates that the COVID-19 pandemic is not over. More vaccine distribution and increased efforts in developing anti-SARS-CoV-2 drugs, social distancing, masking, and practicing good hygiene will be crucial in defeating COVID-19.
\end{abstract}

Keywords: SARS-CoV-2, COVID-19, Angiotensin-converting enzyme 2, Therapeutics, Vaccines

\section{INTRODUCTION}

From what began in Wuhan, China, in December 2019 to one of the worst pandemics of global history affecting millions, the coronavirus disease 2019 (COVID-19) has made its indelible mark. As of July 11, 2021, 186,232,998 active cases and 4,027,858 deaths have been confirmed worldwide. $^{1}$ COVID-19 came from severe acute respiratory syndrome coronavirus-2 (SARS-CoV-2), which belongs to the family Coronaviridae, genus Betacoronavirus, and subgenus Sarbecovirus. ${ }^{2}$ It has been more than a year and a half since SARS-CoV-2 first took a foothold. Yet, there have not been many reliable, proven, and effective anti-SARS-CoV-2 drugs to treat COVID-19 patients. This review provides an overview of COVID-19 and highlights the importance of acquiring an evidence- based treatment to prevent SARS-CoV-2 infection. With the rapid spread of Delta variant, there is a growing need for therapeutics to treat COVID-19 patients as the world continues to face grim consequences.

We utilized PubMed to search literature from January 2018 through June 2021. The studies published in the English language were selected. The words searched to retrieve articles from the database included "COVID-19," "SARSCoV-2," "Treatment," "Remdesivir," "Cytokine Storm," "Hydroxychloroquine," "Epidemiology," "AngiotensinConverting Enzyme 2 Receptor," "Vaccines," and "Mitigation." Preclinical studies, including in vitro and animal model-based literature, searches were limited to the most updated studies. Clinical trials, randomized, placebocontrolled, and double-blind studies were reviewed and 
narrowed down based on most recent dates and large to moderate sample sizes.

The PubMed search identified over 100 publications (publications between 2018 and 2021) on the origin of COVID-19, the modes of transmission, and recent clinical studies done on the effectiveness of specific therapeutics in treating COVID-19 patients. The frequency of studies done on coronavirus increased within the last year due to the outbreak that occurred in 2019. Early studies showed the effectiveness of certain types of vaccines for various viruses. However, the more recent studies focused on SARS-CoV-2, specifically.

\section{DISCUSSION}

The first patient to be infected was a 41-year-old man from Wuhan, China. He was admitted in December 2019 for what was believed to be pneumonia. His social history revealed he worked at a local seafood market. ${ }^{3}$ Soon, the Wuhan Municipal Health Commission announced a "pneumonia outbreak" with an unknown etiology. It was later found out that it was not pneumonia, in fact, it was a SARS-CoV-2 outbreak.

\section{Structure of SARS-CoV-2}

SARS-CoV-2 has a single-stranded, positive-sense ribonucleic acid (RNA) genome that is about 30 kilobases and contains 29,891 base pairs. It is surrounded by an envelope that possesses a viral nucleocapsid. This nucleocapsid has helical symmetry that is atypical in normal positive-sense RNA viruses. The virus has a diameter of 60 to 140 nanometers (nm) and spikes of 9 to $12 \mathrm{~nm}$.

There are four main structural proteins encoded by SARSCoV-2 - spike (S), membrane (M), envelope (E), and nucleocapsid $(\mathrm{N}) .^{4}$ The $\mathrm{S}$ glycoprotein is essential for the virion's entry into the host through the host receptors. ${ }^{5}$ The $\mathrm{S}$ factor can be further categorized into two subunits, S1 and S2. The S1 subunit has two subdomains-the Nterminal domain and a C-terminal domain. ${ }^{6}$ These terminal domains act as receptor-binding domains (RBD).

SARS-CoV-2's genetic sequencing revealed 27 amino acid substitutions in the usual 1,273 amino acid length, six substitutions within the RBD, the site that binds with the angiotensin-converting enzyme-2 (ACE2) receptor. ${ }^{7}$ Sequencing of the initial SARS-CoV-2 virus and the later variants showed 17 nonsynonymous changes within the open reading frames (ORF) 1ab, ORF8, spike gene, and ORF7. These were also present in familial clusters, suggesting evolution of the virus during person-to-person transmission. ${ }^{8}$

The M protein shapes the virion's envelope. Whereas the $\mathrm{E}$ protein plays a critical role in the pathogenesis, assembly, and release of the virus into the host, and the $\mathrm{N}$ protein aids viral genome development outside the host cells. $^{9}$

\section{Phases of transmission}

The COVID-19 epidemic followed five phases of transmission (Figure 1). ${ }^{10}$ Phase one was controlled transmission that occurred through travel to the countries that had infections. Phase two came about through asymptomatic patients with travel history. Local transmission was widespread as SARS-CoV-2 mainly spread to individuals in local areas. Phase three followed soon. It was marked by community transmission. Asymptomatic individuals along with symptomatics started showing COVID-19 symptoms. As SARS-CoV-2 transmission became uncontrollable, phase four started. During this phase, there was intra- and inter-state transmission, resulting in an epidemic. Phase five was finally reached when the outbreak spread to many other countries. $^{10}$ On March 11, 2020, the World Health Organization announced the COVID-19 pandemic. $^{11}$

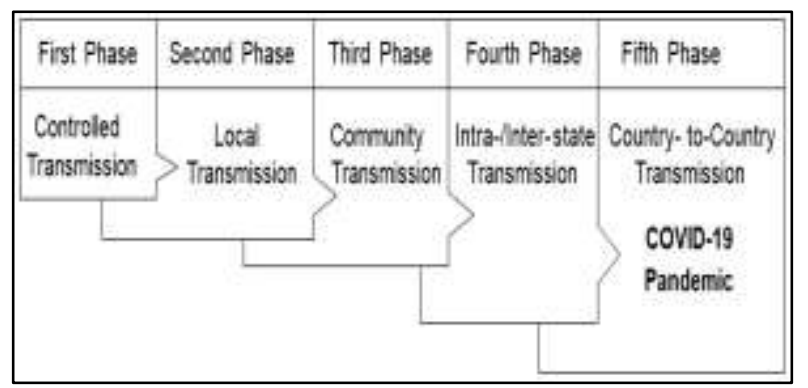

Figure 1: Phases of coronavirus disease 2019 (COVID19) transmission.

\section{Modes of transmission}

SARS-CoV-2 can spread through many ways (Figure 2). The principal mode is through droplets or aerosols when an individual coughs or sneezes onto other people or surfaces. ${ }^{12}$ Fomite transmission is also prevalent. SARSCoV-2 has been shown to live on copper, printing paper, and tissue paper for hours and on cardboards, stainless steel, plastic, treated woods, glass, and banknotes for days. $^{13}$

Another way SARS-CoV-2 can spread is through carrier transmission. ${ }^{14}$ Fecal-oral transmission seems plausible as well, as the SARS-CoV-2 virus was found in stool samples of infected patients. ${ }^{15}$

Pregnancy weakens a woman's immune system, allowing her and the fetus to be easily infected. ${ }^{16}$ A Chinese study demonstrated that neonates born to infected mothers showed temperature instability, tachypnea, grunting, nasal flaring, cough, tachycardia, lethargy, poor feeding, vomiting, diarrhea, and abdominal distention. ${ }^{17}$ 
Another critical postulation is the seasonal transmission. SARS-CoV-2 is most prevalent during the winters and will gradually decrease as the temperature rises. It has been reported that an increase of even one degree Celsius can slow the transmission of SARS-CoV-2 by $13 \% .{ }^{10}$ Although temperature alone would not decrease transmission, mitigation measures, such as social distancing and wearing masks, need to be set to prevent the spread. ${ }^{18}$ One other mode is the sexual transmission. Researchers have detected SARS-CoV-2 in human semen. ${ }^{19}$

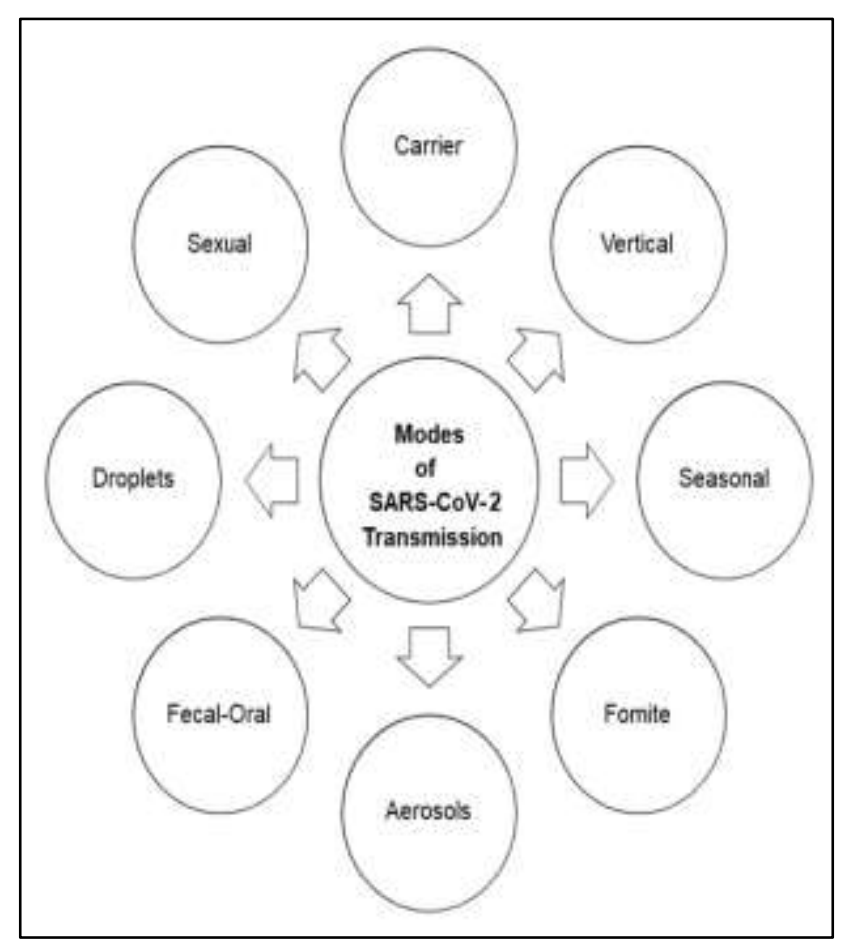

Figure 2: Various modes of severe acute respiratory syndrome coronavirus 2 (SARS-CoV-2) transmission.

\section{Pathogenesis}

The $\mathrm{S}$ protein is the primary determinant of how virulent the SARS-CoV-2 can be. It allows the viral genome to enter the host through the fusion of the host's cellular membrane and the viral membrane. ${ }^{20}$ The $\mathrm{S}$ protein undergoes priming before binding to the host membrane. Specific proteases such as furin, trypsin, trans-membrane protease/serine (TMPRSS), and cathepsins B and L aid the priming. The presence of multiple proteases provides SARS-CoV-2 with the ability to form new variants. ${ }^{21,22}$

It is worth understanding that for the disease to manifest, SARS-CoV-2 must bind to the two key host receptors ACE2 and TMPRSS-2. The ACE2 receptor and TMPRSS2 are co-expressed in specific cells, including macrophages, monocytes, type II pneumocytes, gut enterocytes, and nasal goblet cells of the lung, kidney, and small intestine. Interestingly, children express significantly lower levels of ACE2 and TMPRSS-2 within the upper and lower airways. Consequently, the disease manifests differently when comparing children and adults, with children experiencing a much milder disease outcome. ${ }^{23}$ In addition, chronicled individuals who smoke, have chronic obstructive pulmonary disease, or hypertension have significantly elevated ACE2 and TMPRSS-2 receptors levels, are at risk of developing a severe SARS-CoV-2 infection. ${ }^{24}$

The innate immune system responds to SARS-CoV-2 through alveolar macrophages, neutrophils, monocytes, and dendritic cells inducing systemic inflammation. ${ }^{25}$ Patients were reported to have elevated leukocytes and neutrophil count. ${ }^{26}$ Diabetic patients have increased monocyte levels, thereby, have a greater chance of developing more severe COVID-19. ${ }^{27}$

The adaptative immune system also responds to SARSCoV-2. Patients with moderate to severe COVID-19 were shown to have lymphopenia in peripheral blood. ${ }^{28} \mathrm{~A}$ cluster of differentiation (CD) 4+ T cells induced humoral immunity, while CD8 $+\mathrm{T}$ cells caused the destruction of infected host cells. ${ }^{23}$

Critically, SARS-CoV-2 induces a "cytokine storm" which can adversely impact vital organs. It is characterized by elevated interleukin-1 (IL-1) beta, IL-2, IL-7, IL-8, IL-9, IL-10, IL-17, granulocyte colony-stimulating factor, granulocyte-macrophage colony-stimulating factor, interferon (IFN)-gamma, tumor necrosis factor (TNF)alpha, induced protein-10, monocyte chemoattractant protein-1, and macrophage inflammatory protein-1-alpha and -beta. ${ }^{29}$

\section{Clinical manifestation}

The clinical presentation of COVID-19 typically includes high fever, chills, dry cough, organ dysfunction, fatigue, severe respiratory distress, pulmonary pneumonia, and inflammation. ${ }^{30}$ Patients may experience loss of taste and smell. ${ }^{31}$ Blood chemistry may show leukocytosis, leukopenia with lymphopenia, and hypoalbuminemia. Lactate dehydrogenase, aspartate transaminase, alanine aminotransferase, bilirubin, and D-dimer can be significantly elevated. ${ }^{32}$ The lung biopsy has shown alveolar damage, cellular fibromyxoid exudates, hyaline membrane formation, and pneumocytes desquamation. ${ }^{33}$ The incubation period is between three to six days and carriers can shed SARS-CoV-2 up to three weeks. ${ }^{30}$

\section{Management}

Ever since the COVID-19 broke out in December 2019, the medical community played a catchup game. Globally, we were not prepared to deal with the SARS-CoV-2 as there was very little known about it, let alone the medical treatment. However, in such a short period, there have been remarkable breakthroughs in the therapeutic frontiers and immunization against SARS-CoV-2. Many experimental therapies, drugs, and vaccines showing promise were fasttracked into clinical trials and granted Emergency 
Authorization Use (EUA) by the Food and Drug Administration (FDA).

\section{Recombinant soluble ACE2}

Recombinant ACE2 competitively inhibits SARS-CoV2 's S protein binding with the ACE2 receptors. A phase 2 randomized and double-blind interventional study on human recombinant soluble ACE2 therapy was successful, and the results were submitted to ClinicalTrials.gov on June $7,2021 .^{34}$

\section{Convalescent plasma}

Convalescent plasma was initially used to treat COVID-19 patients due to its ability to bind and inhibit SARS-CoV2's S protein through neutralizing antibodies. ${ }^{35}$ However, findings of a Phase 3 clinical trial in Wuhan, China indicated that the convalescent plasma therapy administered along with standard treatment showed no clinical improvement. ${ }^{36}$

\section{TMPRSS-2 inhibitors}

Mice models have shown that the absence of the TMPRSS2 gene did not completely allow SARS-CoV-2's entry. Camostat mesylate, a TMPRSS-2 inhibitor, partially blocked the entry of SARS-CoV-2 in lung-derived human cell lines. ${ }^{37}$ Still, further clinical research is needed to determine its efficacy.

\section{Hydroxychloroquine}

Hydroxychloroquine, targeting the endocytic pathways of viruses, gained traction early in the pandemic. However, in a large-scale study involving 1,542 patients who were randomly selected to receive Hydroxychloroquine and 3,132 patients under standard care showed no significant clinical benefit of Hydroxychloroquine. ${ }^{38}$

\section{Dexamethasone}

Systemic corticosteroids have been shown to reduce allcause mortality in COVID-19 patients. ${ }^{39}$ For example, six milligrams of dexamethasone once per day for ten days reduced mortality by one-third in ventilated patients with severe COVID-19. ${ }^{40}$

\section{Remdesivir}

Remdesivir is the only drug approved by the FDA to treat hospitalized COVID-19 patients. $^{41}$ It is an adenosine analog that inhibits viral RNA polymerase. A global randomized clinical trial with 1,062 COVID-19 patients showed significant recovery in the treatment (Remdesivir) group (10 days) compared to the placebo group (15 days). Also, the mortality rate in the Remdesivir group was significantly lower $(6.7 \%)$ than the placebo group (11.9\%) by day $15 .^{42}$

\section{Ivermectin}

Ivermectin, is being used in many countries such as India, China, and South Africa for COVID-19. However, the FDA has only approved it as a broad spectrum antiparasitic with antiviral activity. An in vitro study demonstrated that Ivermectin could reduce replication of SARS-CoV-2 in Vero/human signaling lymphocytic activation molecule cells. $^{43}$ Clinical evidence documenting Ivermectin's efficacy in treating COVID-19 is still lacking.

\section{Vaccines}

Vaccines' efficacy, effectiveness, and safety have been quite compelling. There are four types of vaccines being developed: messenger ribonucleic acid (mRNA) vaccines, deoxyribonucleic acid (DNA)-based vaccines, peptidebased vaccines, attenuated or inactivated, and nonreplicating viruses for vaccine generation. ${ }^{44}$

The mRNA vaccines work by administering an mRNA strand that codes for the specific virus. This mRNA, which contains the ORF, is first produced by transcription from a DNA template and RNA polymerase in vitro. ${ }^{44}$ Then the mRNA is carried by liposomes (lipid micro-vesicles). ${ }^{44}$ The ORF within the mRNA serves as an antigen within the host which triggers the host-immune system. The target antigen is represented mainly by the $S$ protein. Examples of mRNA vaccines are Moderna, Pfizer, Abogn, CureVac, and Univ Oxford. ${ }^{45}$

The DNA-based vaccines work through vectors wherein the SARS-CoV-2 gene is transferred to the host to elicit a robust immune response. The engineered non-replicating virus vectors are Chimpanzee adenovirus-AstraZeneca, Gorilla adenovirus-ReiThera, Human adenovirusCanSino, Johnson and Johnson, Acad Mil Med Sci, Gamaleya Res Inst, and Adenovirus for Nasal SprayBeijing Wantai Biol Pharm Enterprise, Acad Mil Sci, Bharat Biotech, AstraZeneca, and Altimmune. ${ }^{45}$

Peptide-based vaccines include a synthetic viral peptide or fusion recombinant peptide that contains the total length or specific domains of the S, M, and N proteins within SARSCoV-2.44 These proteins serve as the antigen against which the body can mount an immune response. Certain vaccines are based on the spike protein plus an adjuvant, including Adimmune, Bektop, Biotechnology Vector, and Clover Biopharmarm, CoVaxx, Inst Finlay de Vacuna Vaccine, Cuba, Medigen, Sanofi, and ZFSW Anhui Zhifei Longcom. ${ }^{45}$

Lastly, SARS-CoV-2 can be attenuated or inactivated to inject the human host and mount a robust immune response. Virus attenuation is achieved by its in vitro replication in unfavorable conditions. Although these vaccines are currently being used in some countries, for example CoronaVac in China and Covaxin in India, there is limited data on their safety and efficacy. ${ }^{45}$ 


\section{Chronic effects}

The COVID-19 pandemic has and will continue to affect millions of people worldwide. A lot is yet to be found about its chronic manifestations. One of the chief concerns that have been highlighted in COVID-19 patients postrecovery following acute infection is fatigue. ${ }^{46}$ In a study, 150 patients were followed up at 7, 30, and 60 days after recovering from COVID-19. After 60 days, $66 \%$ of the adults suffered from anosmia, ageusia, dyspnea, and asthenia. ${ }^{47}$ In addition, some patients were reported to experience alopecia, cognitive and attention deficits, anxiety, insomnia, and depression. ${ }^{48,49}$ More long-term studies are required to thoroughly investigate and understand the physiological, pathological, psychological, and sociological impact of COVID-19 in the future.

\section{CONCLUSION}

There is growing concern that with the Delta variant, the peak of COVID-19 could increase once again. Remdesivir and Dexamethasone seem to provide the most significant clinical benefit. There is hope soon many other therapeutics will be discovered to combat SARS-CoV-2. Social distancing and mask-wearing remain critical in limiting disease spread. In addition, as more vaccines are developed, more people can access and protect themselves with innate and adaptive immunity. However, with that comes the question of equality of access. Economic and financial inequality may impact different countries' ability to manufacture and acquire therapeutics and vaccines, putting their citizens at risk. More research endeavors must be made to determine how access to vaccines affects the transmission of SARS-CoV-2. Viral diseases, such as COVID-19, know no borders. The overcrowding and mixing habitats of humans and animals have brought us to a crossroad that was never imagined. Through sharing knowledge, drugs, and vaccines with severely affected countries, a global alliance must be made to defeat the COVID-19 pandemic.

\section{ACKNOWLEDGEMENTS}

We acknowledge the Learning Resource Center at LECOM Elmira for the support.

Funding: No funding sources

Conflict of interest: None declared

Ethical approval: Not required

\section{REFERENCES}

1. World Health Organization. Weekly operational update on COVID-19. Published July 12, 2021. Available at: https://www.who.int/publications/m/item/weeklyoperational-update-on-covid-19---12-july-2021. Accessed on July 122021.

2. Zhu N, Zhang D, Wang W, Li X, Yang B, Song J, et al. A novel coronavirus from patients with pneumonia in China, 2019. N Engl J Med. 2020;382(8):727-33.

3. Wu F, Zhao S, Yu B, Chen Y-M, Wang W, Song Z$\mathrm{G}$, et al. A new coronavirus associated with human respiratory disease in China. Nature. 2020;579(7798):265-9.

4. dos Santos WG. Impact of virus genetic variability and host immunity for the success of COVID-19 vaccines. Biomed Pharmacother. 2021;136:111272.

5. Valdez-Cruz NA, García-Hernández E, Espitia C, Cobos-Marín L, Altamirano C, Bando-Campos CG, et al. Integrative overview of antibodies against SARS-CoV-2 and their possible applications in COVID-19 prophylaxis and treatment. Microb Cell Factories. 2021;20(1):88.

6. Hu B, Guo H, Zhou P, Shi Z-L. Characteristics of SARS-CoV-2 and COVID-19. Nat Rev Microbiol. 2021;19(3):141-54.

7. Ortiz-Prado E, Simbaña-Rivera K, Gómez- Barreno L, Rubio-Neira M, Guaman LP, Kyriakidis NC, et al. Clinical, molecular, and epidemiological characterization of the SARS-CoV-2 virus and the coronavirus disease 2019 (COVID-19), a comprehensive literature review. Diagn Microbiol Infect Dis. 2020;98(1):115094.

8. Dhama K, Khan S, Tiwari R, Sircar S, Bhat S, Malik YS, et al. Coronavirus disease 2019-COVID-19. Clin Microbiol Rev. 2020;33(4):e00028-20.

9. Machhi J, Herskovitz J, Senan AM, Dutta D, Nath B, Oleynikov MD, et al. The natural history, pathobiology, and clinical manifestations of SARSCoV-2 infections. J Neuroimmune Pharmacol. 2020;15(3):359-86.

10. Kaur S, Bherwani H, Gulia S, Vijay R, Kumar R. Understanding COVID-19 transmission, health impacts and mitigation: timely social distancing is the key. Environ, Dev Sustain. 2021;23(5):6681-97.

11. Revzin MV, Raza S, Warshawsky R, D’Agostino C, Srivastava NC, Bader AS, et al. Multisystem imaging manifestations of COVID-19, part 1: viral pathogenesis and pulmonary and vascular system complications. Radiographics. 2020;40(6):1574-99.

12. Sommerstein R, Fux CA, Vuichard-Gysin D, Abbas M, Marschall J, Balmelli C, et al. Risk of SARSCoV-2 transmission by aerosols, the rational use of masks, and protection of healthcare workers from COVID-19. Antimicrob Resist Infect Control. 2020;9(1):100.

13. van Doremalen $\mathrm{N}$, Bushmaker $\mathrm{T}$, Morris $\mathrm{DH}$, Holbrook MG, Gamble A, Williamson BN, et al. Aerosol and surface stability of SARS-CoV-2 as compared with SARS-CoV-1. N Engl J Med. 2020;382(16):1564-7.

14. Setti L, Passarini F, de Gennaro G, Barbieri P, Perrone MG, Borelli $\mathrm{M}$, et al. SARS-Cov-2RNA found on particulate matter of Bergamo in Northern Italy: first evidence. Environ Res. 2020;188:109754.

15. Ong SWX, Tan YK, Chia PY, Lee TH, Ng OT, Wong MSY, et al. Air, surface environmental, and personal protective equipment contamination by severe acute 
respiratory syndrome coronavirus 2 (SARS-CoV-2) from a symptomatic patient. JAMA. 2020;323(16):1610-2.

16. Wastnedge EAN, Reynolds RM, van Boeckel SR, Stock SJ, Denison FC, Maybin JA, et al. Pregnancy and COVID-19. Physiol Rev. 2021;101(1):303-18.

17. Wang L, Shi Y, Xiao T, Fu J, Feng X, Mu D, et al. Chinese expert consensus on the perinatal and neonatal management for the prevention and control of the 2019 novel coronavirus infection (first edition). Ann Transl Med. 2020;8(3):47.

18. Aboubakr HA, Sharafeldin TA, Goyal SM. Stability of SARS-CoV-2 and other coronaviruses in the environment and on common touch surfaces and the influence of climatic conditions: a review. Transbound Emerg Dis. 2021;68(2):296-312.

19. Li D, Jin M, Bao P, Zhao W, Zhang S. Clinical characteristics and results of semen tests among men with coronavirus disease 2019. JAMA Netw. 2020;3(5):e208292.

20. Hatmal MM, Alshaer W, Al-Hatamleh MAI, Hatmal M, Smadi O, Taha MO, et al. Comprehensive structural and molecular comparison of spike proteins of SARS-CoV-2, SARS-CoV and MERS-CoV, and their interactions with ACE2. Cells. 2020;9(12):2638.

21. Sun J, He W-T, Wang L, Lai A, Ji X, Zhai X, et al. COVID-19: epidemiology, evolution, and crossdisciplinary perspectives. Trends Mol Med. 2020;26(5):483-95.

22. Lokman SM, Rasheduzzaman Md, Salauddin A, Barua R, Tanzina AY, Rumi MH, et al. Exploring the genomic and proteomic variations of SARS-CoV-2 spike glycoprotein: a computational biology approach. Infect Genet Evol. 2020;84:104389.

23. Singh R, Kang A, Luo X. COVID-19: current knowledge in clinical features, immunological responses, and vaccine development. The FASEB Journal. 2021;35(3):e21409.

24. Qiao Y, Wang X-M, Mannan R, Pitchiaya S, Zhang Y, Wotring JW, et al. Targeting transcriptional regulation of SARS-CoV-2 entry factors ACE2 and TMPRSS2. PNAS. 2021;118(1):e2021450118.

25. Hoepel W, Chen H-J, Geyer CE, Allahverdiyeva S, Manz XD, de Taeye SW, et al. High titers and low fucosylation of early human anti-SARS-CoV-2 IgG promote inflammation by alveolar macrophages. Sci Transl Med. 2021;13(596):eabf8654.

26. Qin C, Zhou L, Hu Z, Zhang S, Yang S, Tao Y, et al. Dysregulation of immune response in patients with coronavirus 2019 (COVID-19) in Wuhan, China. Clin Infect Dis. 2020;71(15):762-8.

27. Codo AC, Davanzo GG, Monteiro L de B, de Souza GF, Muraro SP, Virgilio-da-Silva JV, et al. Elevated glucose levels favor SARS-CoV-2 infection and monocyte response through a HIF-1 $\alpha /$ glycolysisdependent axis. Cell Metab. 2020;32(3):437-46.e5.

28. Chen G, Wu D, Guo W, Cao Y, Huang D, Wang H, et al. Clinical and immunological features of severe and moderate coronavirus disease 2019. J Clin Investig. 2020;130(5):2620-9.

29. Huang C, Wang Y, Li X, Ren L, Zhao J, Hu Y, et al. Clinical features of patients infected with 2019 novel coronavirus in Wuhan, China. Lancet. 2020;395(10223):497-506.

30. Dhama K, Patel SK, Pathak M, Yatoo MI, Tiwari R, Malik YS, et al. An update on SARS-CoV-2/COVID19 with particular reference to its clinical pathology, pathogenesis, immunopathology and mitigation strategies. Travel Med Infect Dis. 2020;37:101755.

31. Kumar L, Kahlon N, Jain A, Kaur J, Singh M, Pandey AK. Loss of smell and taste in COVID-19 infection in adolescents. Int $\mathbf{J}$ Pediatr Otorhinolaryngol. 2021;142:110626.

32. Zhao D, Yao F, Wang L, Zheng L, Gao Y, Ye J, et al. A comparative study on the clinical features of coronavirus 2019 (COVID-19) pneumonia with other pneumonias. Clin Infect Dis. 2020;71(15):756-61.

33. Xu Z, Shi L, Wang Y, Zhang J, Huang L, Zhang C, et al. Pathological findings of COVID-19 associated with acute respiratory distress syndrome. Lancet Respir Med. 2020;8(4):420-2

34. Bundgaard H. Recombinant human angiotensinconverting enzyme 2 (rhACE2) as a treatment for patients with COVID-19 (APN01-COVID-19). ClinicalTrials.gov. 2020. Available at: https://clinicaltrials.gov/ct2/show/study/NCT043351 36\#moreinfo. Accessed on 9 June 2021.

35. Brouwer PJM, Caniels TG, van der Straten K, Snitselaar JL, Aldon Y, Bangaru S, et al. Potent neutralizing antibodies from COVID-19 patients define multiple targets of vulnerability. Science. 2020;369(6504):643-50.

36. Li L, Zhang W, Hu Y, Tong X, Zheng S, Yang J, et al. Effect of convalescent plasma therapy on time to clinical improvement in patients with severe and lifethreatening COVID-19. JAMA. 2020;324(5):460.

37. Hoffmann M, Kleine-Weber H, Schroeder S, Krüger $\mathrm{N}$, Herrler T, Erichsen S, et al. SARS-CoV-2 cell entry depends on ACE2 and TMPRSS2 and is blocked by a clinically proven protease inhibitor. Cell. 2020;181(2):271-80.e8.

38. Horby P, Mafham M, Linsell L, Juszczak E, Haynes R, Landray M. Effect of hydroxychloroquine in hospitalized patients with Covid-19. N Engl J Med. 2020;383(21):2030-40.

39. Sterne JAC, Murthy S, Diaz JV, Slutsky AS, Villar J, Angus DC, et al. Association between administration of systemic corticosteroids and mortality among critically ill patients with COVID-19. JAMA. 2020;324(13):1330-41.

40. Rayman G, Lumb AN, Kennon B, Cottrell C, Nagi D, Page E, et al. Dexamethasone therapy in COVID-19 patients: implications and guidance for the management of blood glucose in people with and without diabetes. Diabetic Med. 2021;38(1):e14378.

41. United States Food and Drug Administration. FDA approves first treatment for COVID-19. Published October 22, 2020. Available at: 
https://www.fda.gov/news-events/pressannouncements/fda-approves-first-treatment-covid19. Accessed on 9 June 2021.

42. Beigel JH, Tomashek KM, Dodd LE, Mehta AK, Zingman BS, Kalil AC, et al. Remdesivir for the treatment of Covid-19 - final report. N Engl J Med. 2020;383(19):1813-26.

43. Caly L, Druce JD, Catton MG, Jans DA, Wagstaff KM. The FDA-approved drug ivermectin inhibits the replication of SARS-CoV-2 in vitro. Antivir Res. 2020;178:104787.

44. Marian AJ. Current state of vaccine development and targeted therapies for COVID-19: impact of basic science discoveries. Cardiovasc Pathol. 2021;50:107278.

45. Forni G, Mantovani A. COVID-19 vaccines: where we stand and challenges ahead. Cell Death Differ. 2021;28(2):626-39.

46. Carfì A, Bernabei R, Landi F. Persistent symptoms in patients after acute COVID-19. JAMA. 2020;324(6):603-5.
47. Carvalho-Schneider C, Laurent E, Lemaignen A, Beaufils E, Bourbao-Tournois C, Laribi S, et al. Follow-up of adults with noncritical COVID-19 two months after symptom onset. Clin Microbiol Infect. 2021;27(2):258-63.

48. Xiong Q, Xu M, Li J, Liu Y, Zhang J, Xu Y, et al. Clinical sequelae of COVID-19 survivors in Wuhan, China: a single-centre longitudinal study. Clin Microbiol Infect. 2021;27(1):89-95.

49. Yelin D, Margalit I, Yahav D, Runold M, Bruchfeld J. Long COVID-19-it's not over until? Clin Microbiol Infect. 2021;27(4):506-8.

Cite this article as: Prahalad A, Soti V. Coronavirus disease 2019: an overview. Int J Community Med Public Health 2021;8:5094-100. 